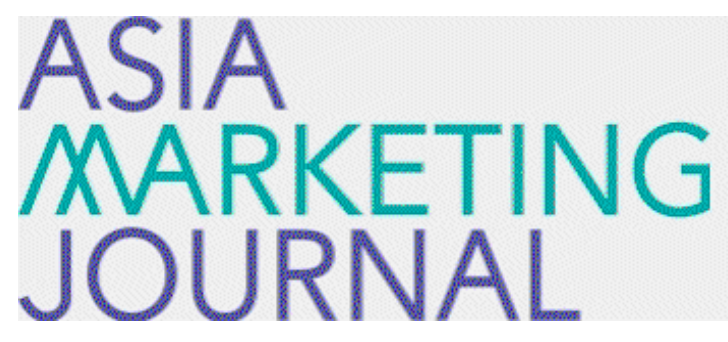

ASIA MARKETING JOURNAL

Volume 17 | Issue 2

Article 4

7-31-2015

\title{
Counting Up while Doing Tasks Makes You Feel More Difficult than Counting Down
}

Hee-Kyung Ahn

Follow this and additional works at: https://amj.kma.re.kr/journal

Part of the Marketing Commons

\section{Recommended Citation}

Ahn, Hee-Kyung (2015) "Counting Up while Doing Tasks Makes You Feel More Difficult than Counting Down," Asia Marketing Journal: Vol. 17 : Iss. 2 , Article 4.

Available at: https://doi.org/10.15830/amj.2015.17.2.63

This Article is brought to you for free and open access by Asia Marketing Journal. It has been accepted for inclusion in Asia Marketing Journal by an authorized editor of Asia Marketing Journal. 


\title{
Counting Up while Doing Tasks Makes You Feel More Difficult than Counting Down*
}

\author{
Hee-Kyung Ahn**
}

\begin{abstract}
In this research, we explore whether mere exposure to external cues with vertical progress (e.g., moving upward or moving downward) can influence individuals' persistence to complete focal tasks. Drawing on the theory of embodied cognition, we propose that, a moving-upward (vs. downward) cue activates the abstract concept of difficulty, which is associated with the physical experience of climbing uphill (vs. downhill). Due to this association between moving uphill and difficulty, merely exposing individuals to the moving-upward cue can induce greater feeling of difficulty and this greater difficulty, in turn, reduce individuals' persistence, compared to exposing individuals to the moving-downward cue. Across three studies, we find supporting evidence for the effect of the external cues with vertical progress on individuals' performance both in physical tasks and in a cognitive task.
\end{abstract}

Key words: Vertical progress, Embodied cognition, Persistence, Motivation, Goal pursuit, Self-regulation

Imagine that you decide to start a new fitness regimen by running thirty minutes every day on a treadmill. The treadmill display allows you to track time in one of two different ways. In one option, the display starts at 00:00 and the time you spend exercising is counted up till it reaches 30:00. In the second option, time is counted down from 30:00 to 0:00. Does the performance of your exercising change as a function of whether time is counted up or counted down? More specifically, is your likelihood of stopping the workout before the 30 minutes has been achieved different across these two conditions? In the present research, we propose that individuals are more likely to quit while counting up because of the meta-

\footnotetext{
* This work was supported by the National Research Foundation of Korea Grant funded by the Korean Government (NRF-2012S1A5A8021974)

** Assistant Professor of Marketing at the School of Business, Hanyang University (hkahn@hanyang.ac.kr)
} 
phoric association between up and difficulty.

The association between moving uphill (downhill) and difficulty (ease) are learnt through physical experiences like ascending or descending stairs, mountains, and hiking trails. All else equal, individuals feel physically more difficult and tired when going up on a hill than when going down on a hill. This is relevant to the fact that one of the lexical definitions of "uphill" is difficult or tiring as a figurative sense. If this figurative language originated from the literal meaning of physical reality, can the mere activation of the figurative meaning "moving upward' induce the feeling of difficulty, thereby reducing individuals' persistence to complete the focal tasks?

The current research tests whether priming external cues with vertical progress can change people's perception about the tasks. In particular, across three studies with differing priming manipulations (i.e., visual and auditory) as well as the type of tasks (i.e., physical and cognitive), we demonstrate the effects of external cues with vertical progress on perception and persistence to complete the tasks.

\section{Motivation, Persistence, and Performance}

Previous research on goals has demonstrated that goal pursuit is driven by both the extent to which the action has been completed, and how much remains (Carver \& Scheier, 1998; Bandura, 1991; Hull, 1932). Highlighting what remains makes individuals pay attention to the lack of actions, thereby increasing motivation (Carver \& Scheier, 1998), whereas highlighting what is completed leads individuals to feel increased sense of self-efficacy, thereby increasing goal pursuit (Bandura, 1991). Further, monitoring individuals' present goal in terms of remaining action (i.e., discrepancies) versus completed action (i.e., past progress) influences the level of aspiration to complete the goal (Koo \& Fishbach, 2010). From a social-developmental perspective, research on learning has demonstrated that motivational determinants affect performance via persistence (Vollmeyer \& Rheinberg, 2000). When enough time is given, highly motivated individuals spend more time in a task than lowly motivated ones (see Atkinson \& Raynor, 1974). In addition, the more time individuals spend learning a task, the better their performances are (Bloom, 1976).

While previous research has heavily documented motivational determinants (e.g., discrepancy, levels of aspiration, and expectancy) and their impact on persistence and performance (Atkinson \& Raynor, 1974; Bloom, 1976; Koo \& Fishbach, 2010; Lewin, 1926; Locke \& Latham, 1990), little is known as to how individuals can be affected by seemingly irrelevant external cues. The current research examines whether and how being exposed to ex- 
ternal cues influence individuals' persistence to carry out focal tasks. Drawing on the theory of embodied cognition, we propose that individuals exposed to moving-upward cues perceive greater difficulty to complete unrelated tasks, and show lower performance in these tasks than individuals exposed to moving-downward cues.

\section{Evidence of Embodiment}

Recent theories of embodied cognition suggest that cognitive representations and operations are fundamentally grounded in their physical context and perception (Barsalou, 1999; Gallese \& Lakoff, 2005; Glenberg, 1997; Lakoff \& Johnson, 1999). Embodiment then refers not only to actual bodily states but also to simulations or reenactments of perceptual, motor, and introspective states that are acquired during experiences with the world, body, and mind (Barsalou 1999, 2008).

Research on metaphors has suggested that some metaphoric associations can be understood through embodied cognition (see Barsalou, 2008). For example, individuals learn a metaphorical association between an abstract concept, "difficulty" and a physical experience, "going uphill" by repeating their physical experiences. Once this metaphorical association has been established, it generalizes across domains (Barsalou, 1999; Lakoff \& Johnson, 1999; Neidenthal et al.,
2005). Consequently, conceptually priming the concept of moving up using different modal stimuli (e.g., counting up, watching a vertically moving-upward progress bar, and listening to a counting-up voice) without the actual physical experience (e.g., going uphill) is enough to activate the abstract concept (e.g., difficulty).

In a similar vein, recent research has demonstrated that a metaphoric relationship between vertical position (up/down) and cardinal direction (north/south) can change people's perception such that people treat figurative language as if it were literal (Nelson \& Simmons, 2009). People believe that it will take longer to go north than south. Moreover, they think that going north is more difficult and effortful than going south due to the metaphoric association between the vertical position (up/down) and the cardinal direction (north/south). Going north does not involve the similar physical elements as people go uphill. However, the association between moving upward and difficulty that people have can make people think of going north as if they are going uphill. We hypothesize that this association between vertical movement and task difficulty (i.e., "uphill is difficult") can influence individuals persistence in irrelevant tasks such as holding a physically demanding pose.

In the following, we test whether priming "uphill" or "moving upward" makes people perceive the given task as more difficult. We vary the modality of manipulations to prime "moving upward" vs. "moving downward" by 
1) having participants count up/down, 2) exposing participants to an upward/downward time progress bar, and 3) using a audio recording of a voice that is counting-up/counting-down.

\section{Study 1: Evidence for the Up-Difficulty Link}

To test our prediction, we first conducted a field study with fifty-five participants of a soccer training program. As a warming up exercise, half of the participants were asked to count numbers up from 1 to 30 and the other half were asked to count numbers down from 30 to 1 as they ran. Right after they finished their exercise, we measured their tiredness, perceived effort in running, and perceived difficulty in running on three 7 -point scales ( $1=$ a lot less tired/effortful/difficult than usual vs. $7=$ a lot more tired/effortful/difficult than usual). We also measured the difficulty of counting the numbers.

Participants who counted the numbers up felt significantly more tired $(M=5.46, S D=$ 1.10 vs. $M=3.14, S D=1.19, t(53)=7.49, p$ $<.001)$, reported having exerted more effort $(M=5.46, S D=1.21$ vs. $M=3.31, S D=$ 1.56, $t(53)=5.67, p<.001)$, and reported greater difficulty $(M=5.96, S D=.92$ vs. $M$ $=3.69, S D=1.29, t(53)=7.47, p<.001)$ than participants who counted the numbers down. The difficulty of counting the numbers were not different between the up and down conditions $(M=4.31, S D=1.32$ vs. $M=$ 4.10, $S D=1.29, t(53)=.58, p=.57)$. These results provide initial evidence for the association between moving upward and difficulty by showing that counting up actually leads individuals to feel greater difficulty than counting down. To more clearly demonstrate the effect of external cues with vertical progress on persistence, we adopted visual time progress bars to prime the concept of moving upward or downward in study 2.

\section{Study 2: A Mediation Analysis}

Study 2 was conducted at a fitness center where a total of sixty-nine participants in a fitness program were randomly assigned to one of two conditions; the upward progress bar and downward progress bar conditions. The upward (downward) progress bars was moving up (down) at a constant velocity over the course of 240 seconds. While watching (one of) the visual time progress bar on a laptop screen, participants were asked to hold a physically demanding pose [in which they had their back to a wall with knees bent at 90 degrees and feet flat on the ground a distance away from the wall] for two minutes. We recorded whether each participant was able to hold the 
pose for 2 minutes. After finishing the task, participants rated how difficult to hold the pose $(1=$ not difficult at all vs. $10=$ very difficult).

A significantly higher percentage of participants in the upward bar condition could not hold the pose ( $\mathrm{p}$ upward $=44 \%$; $\mathrm{p}$ downward $\left.=21 \% ; x^{2}(1)=4.18, p<.05\right)$. The participants in the upward bar condition $(M=7.56$, $S D=1.75)$ reported greater difficulty to hold the pose than those in the downward bar condition $(M=6.58, S D=1.97, t(67)=2.19, p$ $<.05)$.

Importantly, we performed a mediation analysis (Barron \& Kenny, 1986) to examine whether feelings of difficulty mediate the effect of the type of progress bars on the task performance. The upward progress bar reduced participants' persistence to do a physically demanding task $(\beta=1.09, p<.05)$. Moreover, the upward progress bar increased perceived difficulty $(\beta=.98, p<.05)$, which drove the effect on the performance of the task ( $\beta=$ $1.12, p<.001)$. When perceived difficulty was controlled for, the effect of perceived difficulty on persistence remained significant $(\beta=1.10$, $p<.001$ ), but the direct effect of the type of progress bars on the performance was significantly weakened ( $\beta=.79, p=.291$, Sobel test $Z=1.93, p=.05)$. This suggests that perceived difficulty mediates the effect of the type of progress bars on the performance (see figure 1).

Individuals watching the upward progress bar felt greater difficulty than those watching the downward progress bar, which lowered the performance of the focal task.

〈Figure 1〉 Mediation by perceived difficulty. Numbers are unstandardized betas.

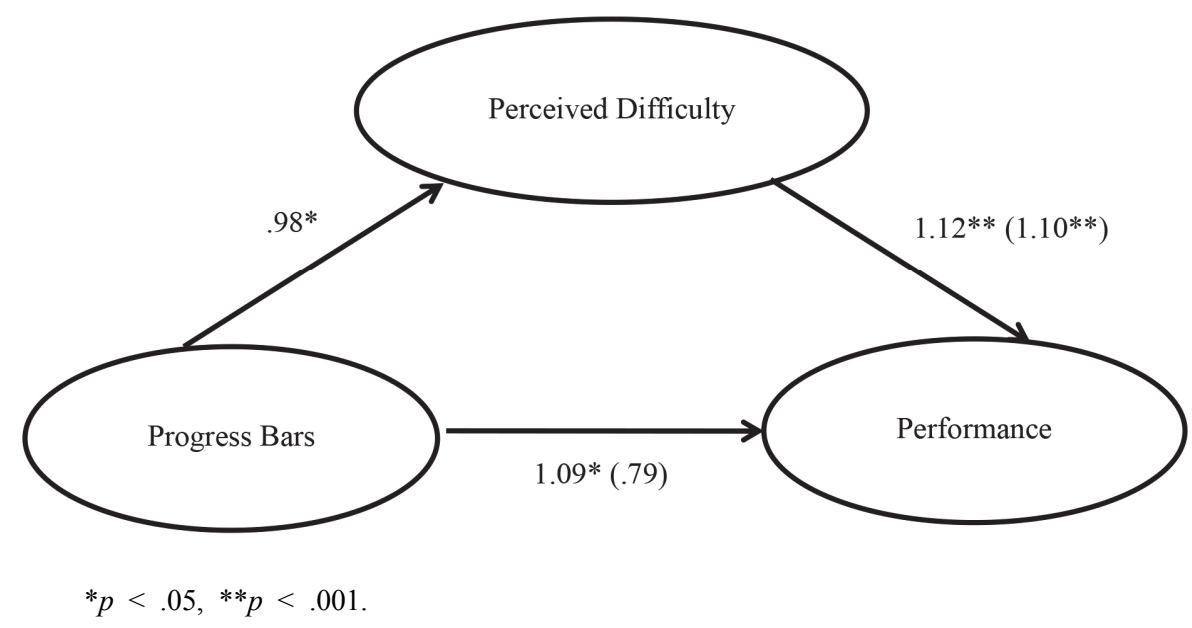




\section{Study 3: From a Physical Task to a Cognitive Task}

We showed thus far that people have an association between moving upward and difficulty in Studies 1 and 2 using physical tasks which differ in terms of the intensity of physical effort. Does the association between moving upward and difficulty apply only to the physical tasks or can it be extended to cognitive tasks? Study 3 tested this possibility. We used auditory progress forms - listening to a counting-up voice vs. listening to a countingdown voice.

Thirty-six participants were randomly assigned to either the counting-up voice or the counting-down voice condition. Participants were asked to solve a maze while the audio recording played in the room. Participants in the counting-up (vs. down) voice condition were told that, while solving the maze, they would hear a voice counting up (vs. down) from 1 to 50 (vs. from 50 to 1), and that they could stop if they could not continue solving the maze before they heard 50 (vs. 1). There was a 5-second interval between every consecutive count, and the total duration of the auditory progress stimuli was 250 seconds. Participants did not know that the maze was actually unsolvable. Our dependent variables were 1) whether they tried to solve the maze until the end or stopped in the middle and 2) how long they tried to solve the maze. In addition, they were asked to how difficult/effortful it was to perform the task on a 7 -point scale $(1=$ not difficulty/effortful at all, $7=$ very difficulty/ effortful). Finally, participants rated their mood and level of arousal on 17-point scales anchored at -8 and 8 (Salovey \& Birnbaum, 1989).

First, a significantly more participants in the counting-up condition (94\%) stopped solving the maze before the end than those in the counting-down condition ( $61 \% ; \mathrm{x}^{2}(1)=5.78$, $p<.05)$. Consistent with the failure rate, participants in the counting-up voice condition ( $M$ $=157.39$ seconds, $S D=57.88$ ) performed the task for significantly less time than those in the counting-down voice condition $(M=197.28$ seconds, $S D=62.07 ; t(34)=-1.99, p=.05)$. In terms of perceived difficulty, participants reported directionally greater difficulty in the counting-down voice condition $(M=4.39, S D$ $=1.42$ ) than in the counting-up voice condition $(M=3.50, S D=1.92 ; t(34)=-1.58$, $p=.12)$. In addition, participants reported greater effort in the counting-down voice condition $(M=5.22, S D=1.26)$ than in the counting-up voice condition $(M=4.44, S D=$ 1.65; $t(34)=-1.59, p=.12)$. Although this finding was unexpected, there could be one explanation. Unknown to participants, the maze we used was unsolvable. The more time participants spent to solve the maze, the more difficult they might have perceived the task to be. Given that we measured perceived diffi- 
culty at the end of the task (not concurrently), participants might have based their judgments on time spent rather than the intensity of effort. Finally, participants' mood and arousal were not significantly different across two conditions (mood: $M_{u p}=3.08, S D=2.72 ; M_{\text {down }}$ $=2.29, S D=2.41 ; t(34)=.93, p=.36$; arousal: $M_{u p}=.51, S D=3.06 ; M_{\text {down }}=.47$, $S D=3.31 ; t(34)=.04, p=.99)$. Study 3 demonstrated that the effect of priming ex$^{-}$ ternal cues with vertical progress on persistence can be extended to a cognitive task.

\section{General Discussion}

Across three studies, we demonstrate that individuals' persistence to carry out focal tasks can be reduced by being exposed to external moving-upward (vs. downward) cues. Further, we show that the perceived difficulty performing the tasks mediates the effect of priming external cues with vertical progress on persistence. Moreover, we replicate this effect in the cognitive task. Drawing on the theory of embodied cognition, we extend previous works on motivation and goals by suggesting that not only internal motivation but also seemingly subtle external cues influence individuals' persistence and performance in the tasks.

We believe that our research will make several important contributions to the burgeoning literature on embodied cognition and goal progress. First, past research on goal pursuits had mainly focused on investigating how consumers monitor their goal pursuit based on both the extent to which the action has been completed, and how much remains. We extend the literature on goal pursuit by establishing that mere exposure of subtle external cues can influence consumers' persistence to complete the tasks. Second, the current research offers a novel contribution to the literature on embodied cognition by demonstrating the effect of association between moving upward and difficulty as to when upward vertical progress decreases individuals' persistence to complete the tasks. Finally, this novel approach has a critical managerial implication in consumers' self control activity with regard to goal pursuit. In other words, many self-control/regulation activities require consumers to set up their relevant goals to accomplish them. In doing so, consumers needs to a certain level of persistence, which can positively influence their goal pursuits and self-control performances. Our research will be able to show clearly that using different external stimuli during consumers' goal pursuit can make difference in acquiring a self-control success. Like the anecdotal episode in the beginning, when people are exposed to the downward movement (or progress), they are better off exercising with less effort.

A limitation of this research is that most of our experiments adopt tasks which require in- 
dividuals' persistence to perform well in the tasks. The type of tasks examined here is characterized by the feature that longer duration typically yields better performance. It would be useful to examine another type of tasks which individuals' persistence may not be critical in performing given tasks well (e.g., accuracy). For example, for certain tasks, people may perform well with a greater accuracy but a shorter duration in conducting the tasks. Given this, future research could be free from the concern that individuals might evaluate perceived difficulty by reflecting the amount of time invested.

Another direction for future research might be to investigate the impact of intrinsic motivation versus extrinsic motivation. Our participants might have extrinsic motivation in conducting the tasks to earn course credits or monetary rewards by following the experimenter's instruction appropriately. Alternatively, they might have very little motivation to finish the tasks. In both cases, we expect the effect of priming subtle external cues on persistence. However, if participants have a strong intrinsic motivation to finish the tasks, the effect of priming the external cues on persistence might be attenuated.

〈Received April 13. 2015〉

〈Revised July 8. 2015〉

$\langle$ Accepted July 15. 2015〉

\section{References}

Atkinson, J. W., \& Raynor, J. O. (1974). Motivation and achievement. Washington, DC: Winston.

Bandura, A. (1991). Self regulation of motivation through anticipatory and self-reactive mechanisms. In R. A. Dienstbier (Ed.), Nebraska Symposium on Motivation, 1990 (pp.69-164). Lincoln, NE: University of Nebraska Press.

Baron, R. M., \& Kenny, D. A. (1986). The moderator-mediator variable distinction in social psychological research: conceptual, strategic and statistical considerations. Journal of Personality and Social Psychology, 51, 1173-1182.

Barsalou, L. W. (1999). Perceptual symbol systems. Behavioral and Brain Sciences, 22, 577-660.

Barsalou, L. W. (2008). Grounded cognition. Annual Review of Psychology, 59, 617-645.

Beilock, S. L. \& Holt, L. E. (2007). Embodied preference judgments: Can likeability be driven by the motor system? Psychological Science, 18, 51-57.

Bloom, B. S. (1976). Human characteristics and school learning. New York, NY: McGrawHill.

Carver, C. S., \& Scheier, M. F. (1998). On the self-regulation of behavior. New York, NY: Cambridge University Press. 
Gallese, V., \& Lakoff, G. (2005). The Brain's concepts: The Role of the sensory-motor system in conceptual knowledge. Cognitive Neuropsychology, 22, 455-479.

Glenberg, A. M. (1997). What Memory is for. Behavioral and Brain Sciences, 20, 1-55.

Hull, C. L. (1932). The goal gradient hypothesis and maze learning. Psychological Review, 39, 25-43.

Koo, M., \& Fishbach, A. (2010). Climbing the goal ladder: How upcoming actions increase level of aspiration. Journal of Personality and Social Psychology, 99, 1-13.

Lakoff, G., \& Johnson, M. (1999). Philosophy in the flesh: The Embodied mind and its challenge to western thought, New York, NY: Basic Books.

Lewin, K. (1926). Intention, will and need. Psychologische Forschuns, 7, 95-121.

Locke, E. A., \& Latham, G. P. (1990). A theory of goal setting and task performance. Upper Saddle River, NJ: Prentice Hall.
Neidenthal, P. M., Barsalou, L. W., Winkielman, P., Krauth-Gruber, S., \& Ric, F. (2005). Embodiment in attitudes, social perception, and emotion. Personality and Social Psychology Review, 9, 184-211.

Nelson, L. D., \& Simmons, J. P. (2009). On southbound ease and northbound fees: Literal consequences of the metaphoric link between vertical position and cardinal direction. Journal of Marketing Research, 46, 715-724.

Salovey, P., \& Birnbaum, D. (1989). Influence of mood on health-relevant cognitions. Journal of Personality and Social Psychology, 57, 539-551.

Sobel, M. E. (1982). Aysmptotic confidence intervals for indirect effects in structural equation models. In S. Leinhardt (Ed.), Sociological methodology (pp. 290-312). San Francisco, CA: Jossey-Bass.

Vollmeyer, R., \& Rheinberg, F. (2000). Does motivation affect performance via persistence? Learning and Instruction, 10, 293-309. 